\title{
Movement velocity in the chair squat is associated with measures of functional capacity and cognition in elderly people at low risk of fall
}

\author{
Carlos Balsalobre-Fernández ${ }^{1,2}$, Ángel Cordón ${ }^{3}$, Nazaret Unquiles ${ }^{3}$ ， Daniel Muñoz-García ${ }^{\text {Corresp. } 4}$ \\ 1 Department of Physical Education, Sport and Human Movement, Universidad Autónoma de Madrid, Madrid, Spain \\ ${ }^{2}$ LFE Research Group, Department of Health and Human Performance, Faculty of Physical Activity and Sport Science-INEF, Universidad Politécnica de \\ Madrid, Madrid, Spain \\ 3 Departamento de Fisioterapia, Universidad Autónoma de Madrid, Centro Superior de Estudios Universitarios La Salle, Madrid, Spain \\ 4 Motion in Brains Research Group, Instituto de Neurociencias y Ciencias del Movimiento, Universidad Autónoma de Madrid, Centro Superior de Estudios \\ Universitarios La Salle, Madrid, Spain \\ Corresponding Author: Daniel Muñoz-García \\ Email address: danimgsan@gmail.com
}

Background. The purpose of this study was to analyze the relationships between muscular performance consisting of a single repetition on the chair squat exercise (CSQ) and different measures of functional capacity, balance, quality of life and cognitive status in older adults. Methods. 40 participants ( 22 women, 18 men; Age=72.2 \pm 4.9 yrs.) joined the investigation. Muscular performance was assessed by measuring movement velocity in the CSQ with no external load using a validated smartphone application (PowerLift for iOS). Functional capacity, balance, quality of life and cognitive status were evaluated using the hand-grip test (HGS), the Berg-scale, the EuroQol 5D (EQ-5D) and the Mini mental state examination questionnaire (MMSE). Finally, participants were divided into two subgroups $(\mathrm{N}=20)$ according to their velocity in the chair squat exercise. Results. Positive correlations were obtained between movement velocity in the CSQ and HGS $(r=0.76, p<$ $0.001)$, the Berg-scale $(r=0.65, p<0.001)$, the EQ-5D $(r=0.34, p=0.03)$ and the MMSE $(r=0.36, p=0.02)$. Participants in the fastest subgroup showed very likely higher scores in the Berg-scale (ES $=1.15$ ) and the HGS (ES = 1.79), as well as likely higher scores in the MMSE scale (ES $=0.69$ ). Discussion. These results could have potential clinical relevance as they support the use of a time-efficient, non-fatiguing test of muscular performance (i.e., the CSQ) to evaluate functional capacity and mental cognition in older adults. 
1

2

3

4

5

6

7 Carlos Balsalobre-Fernández ${ }^{1,2}$, Ángel Cordón ${ }^{3}$, Nazaret Unquiles ${ }^{3}$ \& Daniel Muñoz-García ${ }^{3,4}$

8

$9{ }^{1}$ Department of Physical Education, Sport and Human Movement, Universidad Autónoma de

10

11

12

13

14

15

16

17

18

19

20

21

22

23

\section{AUTHOR COVER PAGE}

\section{MOVEMENT VELOCITY IN THE CHAIR SQUAT IS ASSOCIATED WITH} MEASURES OF FUNCTIONAL CAPACITY AND COGNITION IN ELDERLY PEOPLE AT LOW RISK OF FALL

Madrid, Spain

${ }^{2}$ LFE Research Group, Department of Health and Human Performance. Faculty of Physical Activity and Sport Science-INEF. Universidad Politécnica de Madrid

${ }^{3}$ Departamento de Fisioterapia, Centro Superior de Estudios Universitarios La Salle, Universidad Autónoma de Madrid, Spain

${ }^{4}$ Motion in Brains Research Group, Instituto de Neurociencias y Ciencias del Movimiento, Centro Superior de Estudios Universitarios La Salle, Universidad Autónoma de Madrid, Spain. 


\section{Carlos Balsalobre-Fernández}

25 Department of Physical Education, Sport and Human Movement, Universidad Autónoma de 26 Madrid, Spain

27 LFE Research Group, Department of Health and Human Performance. Faculty of Physical

28 Activity and Sport Science-INEF. Universidad Politécnica de Madrid

29 Address: C/ Fco Tomas y Valiente 3, 28049, Cantoblanco, Madrid, Spain

30 E-mail: carlos.balsalobre@icloud.com

\section{2 Ángel Cordón}

33 Centro Superior de Estudios Universitarios La Salle, Universidad Autónoma de Madrid, Spain.

34 Address: C/ La Salle 10, 28023, Madrid, Spain

35 E-mail: acorta@campuslasalle.es

\section{Nazaret Unquiles}

38 Centro Superior de Estudios Universitarios La Salle, Universidad Autónoma de Madrid, Spain.

39 Address: C/ La Salle 10, 28023, Madrid, Spain

40 E-mail: nunqca@campuslasalle.es

41

42

\section{Daniel Muñoz-García}

44 Departamento de Fisioterapia and Motion in Brains Research Group, Instituto de Neurociencias

45 y Ciencias del Movimiento, Centro Superior de Estudios Universitarios La Salle, Universidad

46 Autónoma de Madrid, Spain. Address: C/ La Salle 10, 28023, Madrid, Spain

47 E-mail: daniel.munoz@lasallecampus.es 


\section{Corresponding author: Daniel Muñoz-García}

53 Departamento de Fisioterapia and Motion in Brains Research Group, Instituto de Neurociencias

54 y Ciencias del Movimiento, Centro Superior de Estudios Universitarios La Salle, Universidad

55 Autónoma de Madrid, Spain. Address: C/ La Salle 10, 28023, Madrid, Spain. E-mail:

56 daniel.munoz@lasallecampus.es

57

58 
59 Movement velocity in the chair squat is associated with measures of functional capacity

60 and cognition in elderly people at low risk of fall

61

62

63

64

65

66

67

68

69

70

71

72

73

74

75

76

77

78

79

80

81

PeerJ reviewing PDF | (2018:02:24957:1:1:NEW 24 Mar 2018) 
82

83

84 ABSTRACT

85 Background. The purpose of this study was to analyze the relationships between muscular 86 performance consisting of a single repetition on the chair squat exercise (CSQ) and different 87 measures of functional capacity, balance, quality of life and cognitive status in older adults.

88 Methods. 40 participants (22 women, 18 men; Age=72.2 \pm 4.9 yrs.) joined the investigation.

89 Muscular performance was assessed by measuring movement velocity in the CSQ with no external 90 load using a validated smartphone application (PowerLift for iOS). Functional capacity, balance, 91 quality of life and cognitive status were evaluated using the hand-grip test (HGS), the Berg-scale, 92 the EuroQol 5D (EQ-5D) and the Mini mental state examination questionnaire (MMSE). Finally, 93 participants were divided into two subgroups $(\mathrm{N}=20)$ according to their velocity in the chair squat 94 exercise.

95 Results. Positive correlations were obtained between movement velocity in the CSQ and HGS $96(r=0.76, \mathrm{p}<0.001)$, the Berg-scale $(r=0.65, \mathrm{p}<0.001)$, the EQ-5D $(r=0.34, \mathrm{p}=0.03)$ and the $97 \operatorname{MMSE}(r=0.36, \mathrm{p}=0.02)$. Participants in the fastest subgroup showed very likely higher scores in 98 the Berg-scale $(\mathrm{ES}=1.15)$ and the HGS $(\mathrm{ES}=1.79)$, as well as likely higher scores in the MMSE 99 scale $(\mathrm{ES}=0.69)$.

100 Discussion. These results could have potential clinical relevance as they support the use of a time101 efficient, non-fatiguing test of muscular performance (i.e., the CSQ) to evaluate functional 102 capacity and mental cognition in older adults. 


\section{INTRODUCTION}

Aging can produce a remarkable decrement on muscle mass, exercise performance and

112 bone mineral density, along many other health-related variables (Evans \& Lexell, 1995; Ruiz et

113 al., 2008; Allison et al., 2013). Specifically, muscular performance has been associated with the

114 risk of suffering falls, which is a strong factor that leads to morbidity or mortality in the elderly

115 (Granacher, Zahner \& Gollhofer, 2008; Volaklis, Halle \& Meisinger, 2015). Resistance training

116 has shown to be a very effective intervention to increase functional capacity, i.e., the ability to

117 perform daily life tasks such as standing up from a chair or walking with proper balance

118 (Granacher, Zahner \& Gollhofer, 2008; Granacher et al., 2013; Marques, Izquierdo \& Pereira, 119 2013). Specifically, the increases in muscle strength and power following a resistance training 120 program are believed to be one of the most important factors that prevent falls and reduces frailty

121 in the elderly (Pijnappels et al., 2008; Granacher et al., 2013; Lopez et al., 2017). Moreover, studies

122 have even shown that progressive resistance training can improve cognitive function in older adults 123 with mild cognitive impairment (Liu-Ambrose, 2010; Gates et al., 2013; Mavros et al., 2017).

124 Several investigations have used different testing protocols to objectively quantify the 125 balance, functional and mental capacities in older adults (like the handgrip strength test -HGS-, 126 the Berg-scale, the chair test or the EuroQol questionnaire -EQ-5D-) in order to develop strategies 127 to prevent falls, reduce frailty and improve the quality of life (Pijnappels et al., 2008; Churchward- 
128 Venne et al., 2015). More recently, smartphone apps have shown to provide accurate measures for 129 different health and performance-related variables in comparison with more expensive laboratory 130 equipment (Bort-Roig et al., 2014; Balsalobre-Fernández, Glaister \& Lockey, 2015; Balsalobre-

131 Fernández et al., 2018). For example, the PowerLift app for iOS devices was shown to provide 132 valid and reliable measures of movement velocity in the squat exercise in comparison with linear 133 transducers (Absolute bias $=-0.005 \pm 0.04 \mathrm{~m} / \mathrm{s} ; \mathrm{SEE}=0.04 \mathrm{~m} / \mathrm{s}$, ICC $>0.9$ ) (Balsalobre-Fernández 134 et al., 2017). Finally, during the last decade, the measurement of movement velocity in tasks like 135 the squat has been proposed as a reliable method to quantify muscular performance(Conceição et 136 al., 2016; Muñoz-López et al., 2017; García-Ramos et al., 2017a; Balsalobre-Fernández, García-

137 Ramos \& Jiménez-Reyes, 2017); in this sense, it is known that, for a certain mass, higher 138 movement velocity results in greater force and power production (Samozino et al., 2008; Muñoz139 López et al., 2017). Taking into account that muscular performance has been proven to be related 140 with functional capacity and mental cognition(Pereira et al., 2012; Marques, Izquierdo \& Pereira, 141 2013; Mavros et al., 2017; Lopez et al., 2017) and considering that studies have shown that mean 142 velocity is a reliable measure of muscular performance(Bazuelo-Ruiz et al., 2015; Muñoz-López 143 et al., 2017; García-Ramos et al., 2017b), it is reasonable to think that the analysis of movement

144 velocity during functional tasks in elder populations (such as standing up from a chair) could have 145 relevant clinical applications. However, to the best of our knowledge, no studies have analyzed 146 movement velocity in tasks like the chair squat (from now on, CSQ) in older adults, nor have 147 investigated its potential associations with measures of balance, functional capacity and cognition. 148

For this, the aim of this study was to analyze if mean task velocity of a single repetition on 150 the CSQ using a smartphone app is related to other widespread balance, functional and cognitive 
151 tests in older adults at low risk of falling. Our hypothesis is that velocity in the chair squat will be

152 related with several measures of strength, balance and cognition.

153

154 MATERIAL \& METHODS

155 Experimental approach to the problem

156 This investigation is a correlational study with parallel groups comparison. Performance in

157 functional capacity tests (as well as scores in cognition and mental questionnaires were correlated

158 with mean velocity in the chair squat task (CSQ). Participants were also divided into two groups

159 based on their performance in the chair squat test for comparison purposes.

160

161 Participants'

162 Forty participants from the same nursing home who met the eligibility criteria were recruited for

163 this study $\left(\mathrm{n}=40 ; 22\right.$ women, 18 men; Age $=72.2 \pm 4.9$ yrs.; Body mass index $\left.=27.8 \pm 3.3 \mathrm{~kg} / \mathrm{m}^{2}\right)$.

164 Eligibility criteria were established as follow: 1) age between 65-85 yrs. old; 2) not having joined

165 any resistance training program at least in the past 5 years; 3) not having any diagnosed 166 musculoskeletal disorder or injury; 4) being categorized at low risk of falling in the Berg scale.

167 The Spanish version of the Yale Physical Activity Questionnaire -Y-PAQ- (Katz et al., 2014)

168 was administered to evaluate participant's basal levels of physical activity, who scored $52.7 \pm 23.2$

169 points. According to this questionnaire, participants with a score of 51 points or less are 170 categorized as sedentary.

171 The study protocol complied with the Declaration of Helsinki for Human Experimentation and

172 was approved by the ethics committee at the institutional review board (CSEULS-PI-059/2015).

173 Written informed consent was obtained from each subject before participation. 
175 Procedures

176 During a single morning of testing conducted in their nursing home (approximately, from

$17710 \mathrm{am}$ to $14 \mathrm{am}$ ), participants performed several tasks to test their balance and functional capacities.

178 No warm-up was conducted prior to these tasks. First, the hand grip strength (HGS, in $\mathrm{kg}$ ) was

179 tested with a hydraulic dynamometer (Lafayette Instrument Evaluation, USA) as reported in the 180 literature: participants hold the dynamometer with the wrist in a neutral position and the elbow 181 fully extended (Pereira et al., 2012). If the participant supinated their wrist or flexed their elbow, 182 the measurement was repeated. Two attempts were conducted with each hand, and the final score 183 was calculated as the average value of the best attempt of each hand. Second, the Berg-scale was 184 calculated as the sum of scores of 14 different balance and functional tasks as described elsewhere 185 (Berg et al., 1992; Fernandez-Alonso, Muñoz-García \& Touche, 2016). Scores in the Berg-scale 186 were categorized as follows: high risk of falling (0-20), moderate risk (21-40) and low risk (>41) 187 (Fernandez-Alonso, Muñoz-García \& Touche, 2016). Third, the Yale Physical Activity (Y-PAQ) 188 (Katz et al., 2014), the EuroQol (EQ-5D component) and the Mini mental state examination 189 (MMSE) (Cano Gutiérrez et al., 2016) questionnaires were administrated to evaluate levels of 190 daily physical activity, quality of life and cognitive status, respectively.

191

192 193 194 195 196

Finally, we asked the participants to perform a chair squat (CSQ): a modification of the squat exercise in which the task starts with the participant sat in a chair. We instructed the participants to sit on a chair (which was the same for every participant) without touching its backrest with their back, to place their feet flat on the ground and at the width of their hips, to keep their arms crossed in their chest and to stand up as fast as possible until the knees were fully 
197 extended, and the trunk was in an upright position. Experimented researchers and certified strength 198 and conditioning specialists carefully observed every attempt and asked the participants to repeat 199 the CSQ in case the aforementioned criteria for a valid attempt were not met. See Figure 1 for 200 more details. We measured mean velocity (in $\mathrm{m} / \mathrm{s}$ ) of 4 attempts (separated by 10 seconds of 201 passive rest) using a validated app (PowerLift for iOS, v.5.4) (Balsalobre-Fernández et al., 2018) 202 installed on an iPhone 6 running iOS 10.3 .3 (Apple Inc., USA). To measure mean velocity, 203 PowerLift uses the well-known Newtonian equation (1):

204

$205 v=d / t$

206

207 where $v$ is the mean velocity (in $\mathrm{m} / \mathrm{s}$ ), $d$ (in $\mathrm{m}$.) the range of motion of the movement (in this case, 208 the difference between the height of the participants standing and sitting on a chair) and $t$ the time 209 (in s.) of the task, which was calculated by the app as the time between two frames selected by the 210 user. The beginning of the task was considered as the first frame in which the participant took-off 211 the chair, and the end of the task was considered as the first frame in which the knees were 212 completely extended. To record the videos, a researcher held the iPhone on his hand in portrait 213 position and recorded each lift from the side of the participant at $1.5 \mathrm{~m}$ from the chair in order to 214 see the full range of motion as close as possible. The height of the participants, both in the standing 215 and sitting position, was measured using a wall-mounted stadiometer (Seca, Germany). The best 216 of the four attempts was used for the statistical analyses. 

included the half who reached higher velocities in the test, while G2 included the other half of 220 participants.

221

222 Statistical analyses

223 We used the Pearson's product-moment correlation coefficient to calculate the association between 224 movement velocity in the CSQ and the functional measures. The level of significance was set at $225 \mathrm{p}<0.05$. To compare groups, we used standardized mean differences (SMD) with the 226 corresponding 90\% confidence interval as proposed by Hopkins et al. (Hopkins et al., 2009). The 227 criteria for interpreting the magnitude of the SMD were: trivial $(<0.2)$, small $(0.2-0.6)$, moderate 228 (0.6-1.2), large (1.2-2.0), and extremely large $(>2.0)$. Quantitative chances of better or worse 229 scores were assessed qualitatively as follows: $<1 \%$, almost certainly not; $1-5 \%$, very unlikely; 5$23025 \%$, unlikely; 25-75\%, possible; 75-95\%, likely; 95-99\%, very likely; and >99\%, almost certain.

231 If the changes of better or worse were both $>5 \%$, the true difference was assessed as unclear. 232

\section{RESULTS}

\section{Correlation between variables}

236 The mean velocity in the chair squat was positively and significantly correlated with the HGS ( $r=$ $2370.76, \mathrm{p}=0.00)$, the Berg-scale $(r=0.65, \mathrm{p}=0.00)$, the Y-PAQ $(r=0.34, \mathrm{p}=0.03)$, the EQ-5D $(r$ $238=0.34, \mathrm{p}=0.03)$ and the MMSE scale $(r=0.36, \mathrm{p}=0.02)$. See Figure 2 for more details. 
241 Participants in G1 showed very likely higher scores in the Berg-scale (99/1/0; SMD = 1.15, 90\%

$242 \mathrm{CI}=0.51-1.18)$ and the HGS $(100 / 0 / 0 ; \mathrm{SMD}=1.79,90 \% \mathrm{CI}=0.98-2.59)$, as well as likely higher

243 scores in the MMSE scale $(87 / 10 / 3 ; \mathrm{SMD}=0.69,90 \% \mathrm{CI}=-0.04-1.42, \mathrm{p}<0.05)$ and the Y-PAQ

$244(93 / 5 / 2 ; \mathrm{SMD}=0.87,90 \% \mathrm{CI}=0.13-1.61, \mathrm{p}<0.01)$. No other variable showed meaningful,

245 statistically significant differences between G1 and G2. See Figure 3 for more details.

247 DISCUSSION

248 The main finding of this study was the moderate to large positive correlations observed 249 between mean velocity in the chair squat and both the hand grip strength and the Berg-scale. These 250 results are in line with previous research that showed remarkable associations between the 30-s. 251 chair test (i.e., completing as many chair squat repetitions as possible within 30 s.) and other 252 measures of functional capacity (Churchward-Venne et al., 2015; Bongers et al., 2015). In this 253 sense, several studies have showed that increased levels of lower-limbs muscular performance 254 (which traditionally has been tested measuring the times that the participant could sit and stand on 255 a chair in 30-s) are strongly associated with lower risk of falls(Pijnappels et al., 2008; Churchward256 Venne et al., 2015; Lopez et al., 2017). However, the CSQ test described in our study could be a 257 proposed as a faster, less fatiguing test for the measurement for older adults since it only requires 258 a single repetition.

We observed moderate to large differences between groups for both the hand grip strength 260 and the Berg-scale, with participants in the strong group (G1) having likely to very likely higher 261 scores than their counterparts from G2. Muscular strength seems to be an extremely important 262 factor to prevent falls and increase functional capacity in the elderly, and recent studies have even 263 shown that there is an inverse relationship between strength and mortality (Pijnappels et al., 2008; 
264 Granacher et al., 2013; Volaklis, Halle \& Meisinger, 2015; Stamatakis et al., 2017; Lopez et al., 265 2017). During the last decade, movement velocity in resistance exercises (such as the back squat) 266 has been proposed to be a very reliable metric to monitor muscular strength since, for a certain 267 load, higher movement velocity means having higher maximal strength and power (Gonzalez268 Badillo \& Sánchez-Medina, 2010; Muñoz-López et al., 2017; García-Ramos et al., 2017b). In fact, 269 it has been demonstrated that high-speed power training is an effective exercise approach leading 270 to large gains in muscle performance and functional capacity(Pereira et al., 2012; Marques, 271 Izquierdo \& Pereira, 2013). Therefore, in order to better analyze the changes in muscular 272 performance of older participants after a strength/power training program, the measurement of 273 movement velocity in tasks like the chair test or the CSQ could have important clinical 274 applications. However, its use among elder populations is still poorly investigated. To the best of 275 our knowledge, this is the first study analyzing mean velocity in the chair squat exercise as a mean 276 to monitor muscular performance in a geriatric population.

277 Moreover, our results showed a significant correlation between the MMSE cognition test and movement velocity in the CSQ, as well as likely moderate higher scores in the MMSE in the strongest group of participants. Recent research has observed that muscular strength has a positive effect on cognition as, for example, it has been showed that a progressive resistance training program produces a significant improvement in the cognitive function in individuals with mild cognitive impairment (Mavros et al., 2017). It seems that some of the main factors that could explain the beneficial effects of resistance training in cognition are the reduction on serum homocysteine and the increased concentrations of insulin-like growth factor I that this type of training produce, which has been associated with impaired cognitive performance or increased 
287 neural growth, respectively(Liu-Ambrose \& Donaldson, 2008). Therefore, our results are

288 consistent with previous research and highlights the relationship between muscular performance

289 and cognition in elders, since there was a significant trend by which participants with higher

290 velocity in the CSQ had better scores in the MMSE cognition test.

291

292

However, this study has two main limitations that should be considered when trying to 293 replicate it: first, the average age of the participants was about $72 \mathrm{yrs}$. and, therefore, conclusions 294 should not be generalized to older geriatric populations. Finally, participants in our study had a 295 mean score in the Berg-scale of 53.3 points, which is considered as "low risk" of falling. Again, 296 conclusions should be taken with precaution as other geriatric populations at higher risks of having falls were not studied in our investigation. These results might help strength and conditioning 298 coaches and physical therapists working with elder populations to monitor muscular performance in a time-efficient, effort-less, affordable way within their fall-prevention protocols.

300

301

302

CONCLUSIONS

This study showed that the mean velocity recorded by a smartphone app during the chair squat exercise is positively related to measures of functional performance, balance and cognition 304 in elders.

305

306

307

308

309

\section{REFERENCES}

Allison SJ., Folland JP., Rennie WJ., Summers GD., Brooke-Wavell K. 2013. High impact exercise increased femoral neck bone mineral density in older men: a randomised unilateral intervention. Bone 53:321-328. DOI: 10.1016/j.bone.2012.12.045. 
310 Balsalobre-Fernández C., García-Ramos A., Jiménez-Reyes P. 2017. Load-velocity profiling in 311 the military press exercise: Effects of gender and training. International Journal of Sports

312

313

314

315

316

317

318

319

320

321

322

323

324

325

326

327

328

329

330

331

332 Science \& Coaching 0:174795411773824. DOI: 10.1177/1747954117738243.

Balsalobre-Fernández C., Glaister M., Lockey RA. 2015. The validity and reliability of an iPhone app for measuring vertical jump performance. Journal of Sports Sciences 33:15741579. DOI: $10.1080 / 02640414.2014 .996184$.

Balsalobre-Fernández C., Marchante D., Baz-Valle E., Alonso-Molero I., Jiménez SL., MuñózLópez M. 2017. Analysis of Wearable and Smartphone-Based Technologies for the Measurement of Barbell Velocity in Different Resistance Training Exercises. Frontiers in Physiology 8. DOI: 10.3389/fphys.2017.00649.

Balsalobre-Fernández C., Marchante D., Muñoz-López M., Jiménez SL. 2018. Validity and reliability of a novel iPhone app for the measurement of barbell velocity and 1RM on the bench-press exercise. Journal of Sports Sciences 36:64-70. DOI:

10.1080/02640414.2017.1280610.

Bazuelo-Ruiz B., Padial P., García-Ramos A., Morales-Artacho AJ., Miranda MT., Feriche B. 2015. Predicting maximal dynamic strength from the load-velocity relationship in squat exercise. Journal of strength and conditioning research 29:1999-2005. DOI: 10.1519/JSC.0000000000000821.

Berg KO., Wood-Dauphinee SL., Williams JI., Maki B. 1992. Measuring balance in the elderly: validation of an instrument. Canadian journal of public health 83 Suppl 2:S7-11.

Bongers KTJ., Schoon Y., Graauwmans MJ., Hoogsteen-Ossewaarde ME., Rikkert MGMO. 2015. Safety, Feasibility, and Reliability of the Maximal Step Length, Gait Speed, and Chair Test Measured by Seniors Themselves: The Senior Step Study. Journal of Aging and 
Physical Activity 23:438-443. DOI: 10.1123/japa.2013-0231.

334 Bort-Roig J., Gilson ND., Puig-Ribera A., Contreras RS., Trost SG. 2014. Measuring and

335 Influencing Physical Activity with Smartphone Technology: A Systematic Review. Sports

336 Medicine 44:671-686. DOI: 10.1007/s40279-014-0142-5.

337 Cano Gutiérrez C., Arciniegas Rubio A., Germán Borda M., Samper-Ternent R., Gil Laverde F., 338 Londoño Trujillo D. 2016. Perception of health-related quality of life using the EURO-QOL 339 in older adults in Bogotá, Colombia. European Geriatric Medicine 7:340-345. DOI: $340 \quad 10.1016 /$ j.eurger.2016.01.008.

341 Churchward-Venne TA., Tieland M., Verdijk LB., Leenders M., Dirks ML., de Groot LCPGM., 342 van Loon LJC. 2015. There Are No Nonresponders to Resistance-Type Exercise Training in Older Men and Women. Journal of the American Medical Directors Association 16:400411. DOI: 10.1016/j.jamda.2015.01.071.

Conceição F., Fernandes J., Lewis M., Gonzaléz-Badillo JJ., Jimenéz-Reyes P. 2016. Movement velocity as a measure of exercise intensity in three lower limb exercises. Journal of Sports Sciences 34:1099-1106. DOI: 10.1080/02640414.2015.1090010.

Evans WJ., Lexell J. 1995. Human Aging, Muscle Mass, and Fiber Type Composition. The Journals of Gerontology Series A: Biological Sciences and Medical Sciences 50A:11-16. DOI: 10.1093/gerona/50A.Special_Issue.11.

351 Fernandez-Alonso L., Muñoz-García D., Touche R La. 2016. The level of physical activity 352 affects the health of older adults despite being active. Journal of Exercise Rehabilitation 353 12:194-201. DOI: 10.12965/jer.1632566.283.

354 García-Ramos A., Haff GG., Pestaña-Melero FL., Pérez-Castilla A., Rojas FJ., Balsalobre355 Fernández C., Jaric S. 2017a. Feasibility of the Two-Point Method for Determining the 
356 One-Repetition Maximum in the Bench Press Exercise. International Journal of Sports 357 Physiology and Performance:1-23. DOI: 10.1123/ijspp.2017-0374.

358 García-Ramos A., Pestaña-Melero FL., Pérez-Castilla A., Rojas FJ., Haff GG. 2017b. Mean

359 velocity vs. mean propulsive velocity vs. peak velocity. Journal of Strength and

360 Conditioning Research:1. DOI: 10.1519/JSC.0000000000001998.

361 Gates N., Singh MAF., Sachdev PS., Valenzuela M. 2013. The effect of exercise training on 362 cognitive function in older adults with mild cognitive impairment: A meta-analysis of 363 randomized controlled trials. American Journal of Geriatric Psychiatry 21:1086-1097. $364 \quad$ DOI: $10.1016 /$ j.jagp.2013.02.018.

365 Gonzalez-Badillo JJ., Sánchez-Medina L. 2010. Movement Velocity as a Measure of Loading 366 Intensity in Resistance Training. International Journal of Sports Medicine 31:347-352.

367 Granacher U., Gollhofer A., Hortobágyi T., Kressig R., Muehlbauer T. 2013. The Importance of 368 Trunk Muscle Strength for Balance, Functional Performance, and Fall Prevention in 369 Seniors: A Systematic Review. Sports Medicine 43:627-641.

370 Granacher U., Zahner L., Gollhofer A. 2008. Strength, power, and postural control in seniors:

371 Considerations for functional adaptations and for fall prevention. European Journal of Sport 372 Science 8:325-340. DOI: 10.1080/17461390802478066.

373 Hopkins WG., Marshall SW., Batterham AM., Hanin J. 2009. Progressive Statistics for Studies 374 in Sports Medicine and Exercise Science. Medicine \& Science in Sports \& Exercise 41:337512.

376 Katz JN., Perez MT., Niu NN., Dong Y., Brownlee SA., Elman SA., Stenquist DS., Santiago A., 377 Sanchez ES., Collins JE. 2014. Development and validation of a Spanish translation of the $378 \quad$ Yale activity questionnaire. BMC Musculoskeletal Disorders 15:120. DOI: 10.1186/1471- 
379

380

381

382

383

384

385

386

387

388

389

390

391

392

393

394

395

396

397

398

399

400

401

2474-15-120.

Liu-Ambrose T. 2010. Resistance Training and Executive Functions. Archives of Internal Medicine 170:170. DOI: 10.1001/archinternmed.2009.494.

Liu-Ambrose T., Donaldson MG. 2008. Exercise and cognition in older adults: is there a role for resistance training programmes? British Journal of Sports Medicine 43:25-27. DOI:

$$
\text { 10.1136/bjsm.2008.055616. }
$$

Lopez P., Pinto RS., Radaelli R., Rech A., Grazioli R., Izquierdo M., Cadore EL. 2017. Benefits of resistance training in physically frail elderly: a systematic review. Aging Clinical and Experimental Research 0:0. DOI: 10.1007/s40520-017-0863-z.

Marques MC., Izquierdo M., Pereira A. 2013. High-Speed Resistance Training in Elderly People: A New Approach Toward Counteracting Age-Related Functional Capacity Loss. Strength \& Conditioning Journal 35:23-29.

Mavros Y., Gates N., Wilson GC., Jain N., Meiklejohn J., Brodaty H., Wen W., Singh N., Baune BT., Suo C., Baker MK., Foroughi N., Wang Y., Sachdev PS., Valenzuela M., Fiatarone Singh MA. 2017. Mediation of Cognitive Function Improvements by Strength Gains After Resistance Training in Older Adults with Mild Cognitive Impairment: Outcomes of the Study of Mental and Resistance Training. Journal of the American Geriatrics Society 65:550-559. DOI: 10.1111/jgs.14542.

Muñoz-López M., Marchante D., Cano-Ruiz MA., Chicharro JL., Balsalobre-Fernández C. 2017. Load-, Force-, and Power-Velocity Relationships in the Prone Pull-Up Exercise. International Journal of Sports Physiology and Performance 12:1249-1255. DOI: 10.1123/ijspp.2016-0657.

Pereira A., Izquierdo M., Silva AJ., Costa AM., Bastos E., Gonzalez-Badillo JJ., Marques MC. 
402 403 404 405 406 407 408 409 410

2012. Effects of high-speed power training on functional capacity and muscle performance in older women. Experimental gerontology 47:250-255. DOI: 10.1016/j.exger.2011.12.010.

Pijnappels M., van Burg JCE., Reeves ND., van Dieën JH. 2008. Identification of elderly fallers by muscle strength measures. European Journal Of Applied Physiology 102:585-592.

Ruiz JR., Sui X., Lobelo F., Morrow JR., Jackson AW., Sjostrom M., Blair SN. 2008. Association between muscular strength and mortality in men: prospective cohort study. BMJ 337:a439-a439. DOI: 10.1136/bmj.a439.

Samozino P., Morin J-B., Hintzy F., Belli A. 2008. A simple method for measuring force, velocity and power output during squat jump. Journal of Biomechanics 41:2940-2945. DOI: 10.1016/j.jbiomech.2008.07.028.

Stamatakis E., Lee I-M., Bennie J., Freeston J., Hamer M., O’Donovan G., Ding D., Bauman A., Mavros Y. 2017. Does strength promoting exercise confer unique health benefits? A pooled analysis of eleven population cohorts with all-cause, cancer, and cardiovascular mortality endpoints. American Journal of Epidemiology. DOI: 10.1093/aje/kwx345.

Volaklis KA., Halle M., Meisinger C. 2015. Muscular strength as a strong predictor of mortality: A narrative review. European Journal of Internal Medicine 26:303-310. DOI: 10.1016/j.ejim.2015.04.013.

\section{FIGURES CAPTIONS}

Figure 1. Initial (A) and final (B) position during the chair squat exercise (CSQ). 
424 Figure 2. Correlation between mean velocity (in $\mathrm{m} / \mathrm{s}$ ) in the chair squat exercise and A) the Mini 425 mental state examination test (MMSE, black dots) and the Yale Physical Activity Questionnaire 426 (Y-PAQ, white diamonds), and B) the hand grip strength (HGS, black dots) and the Berg-scale 427 (white diamonds).

428

429 Figure 3. Standardized mean differences (with 90\% CI) between scores for the hand grip strength 430 (HGS), the EQ-5D scale, the Yale Physical Activity Questionnaire (Y-PAQ), the Mini mental state 431 examination (MMSE) and the Berg scale from the strongest (G1) and weaker (G2) participants. 


\section{Figure 1}

Figure 1

Initial (A) and final (B) position during the chair squat exercise (CSQ).

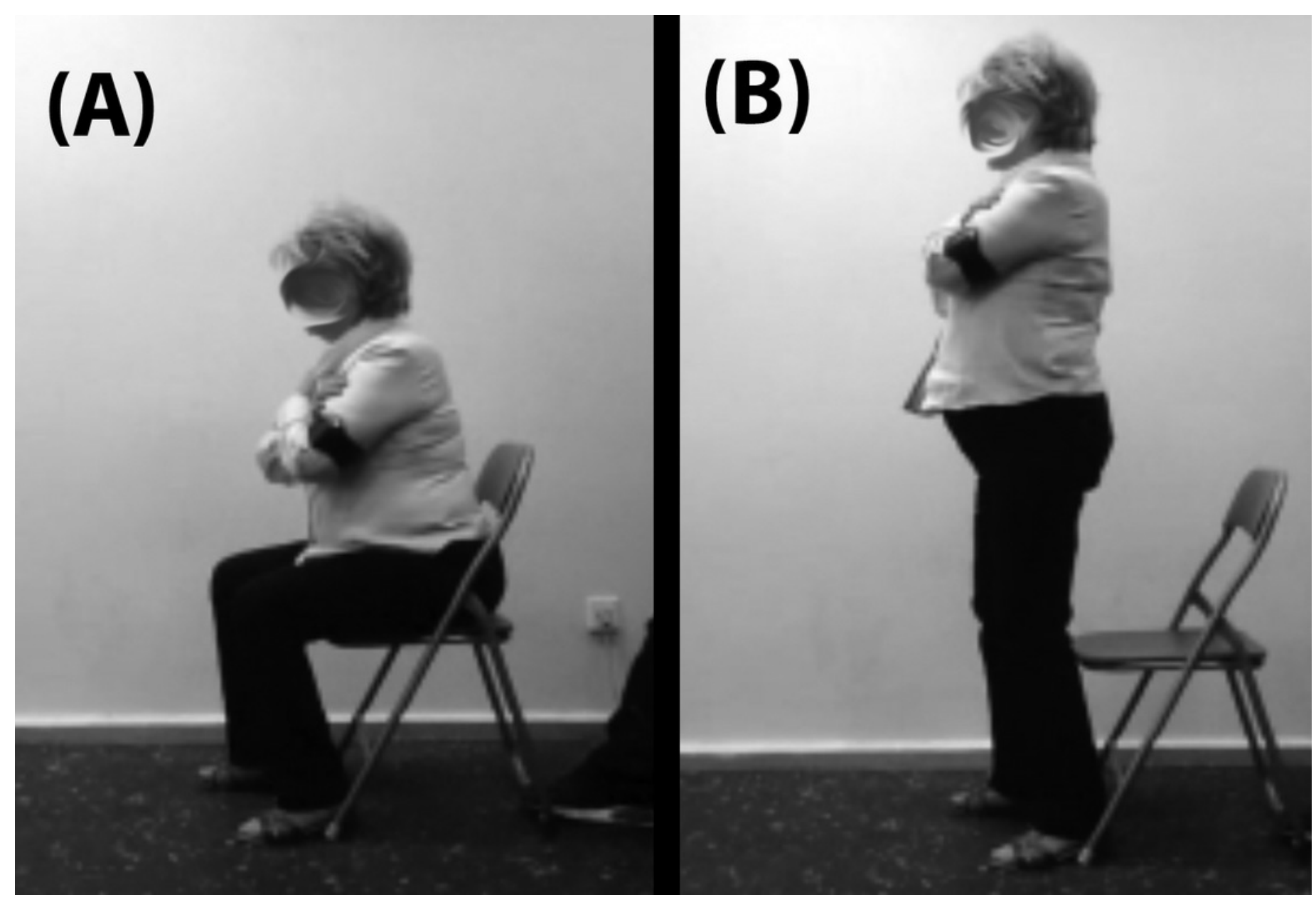




\section{Figure 2}

Figure 2

Correlation between mean velocity (in $\mathrm{m} / \mathrm{s}$ ) in the chair squat exercise and A) the Mini mental state examination test (MMSE, black dots) and the Yale Physical Activity Questionnaire (YPAQ, white diamonds), and B) the hand grip strength (HGS, black dots) and the Berg-scale (white diamonds). 

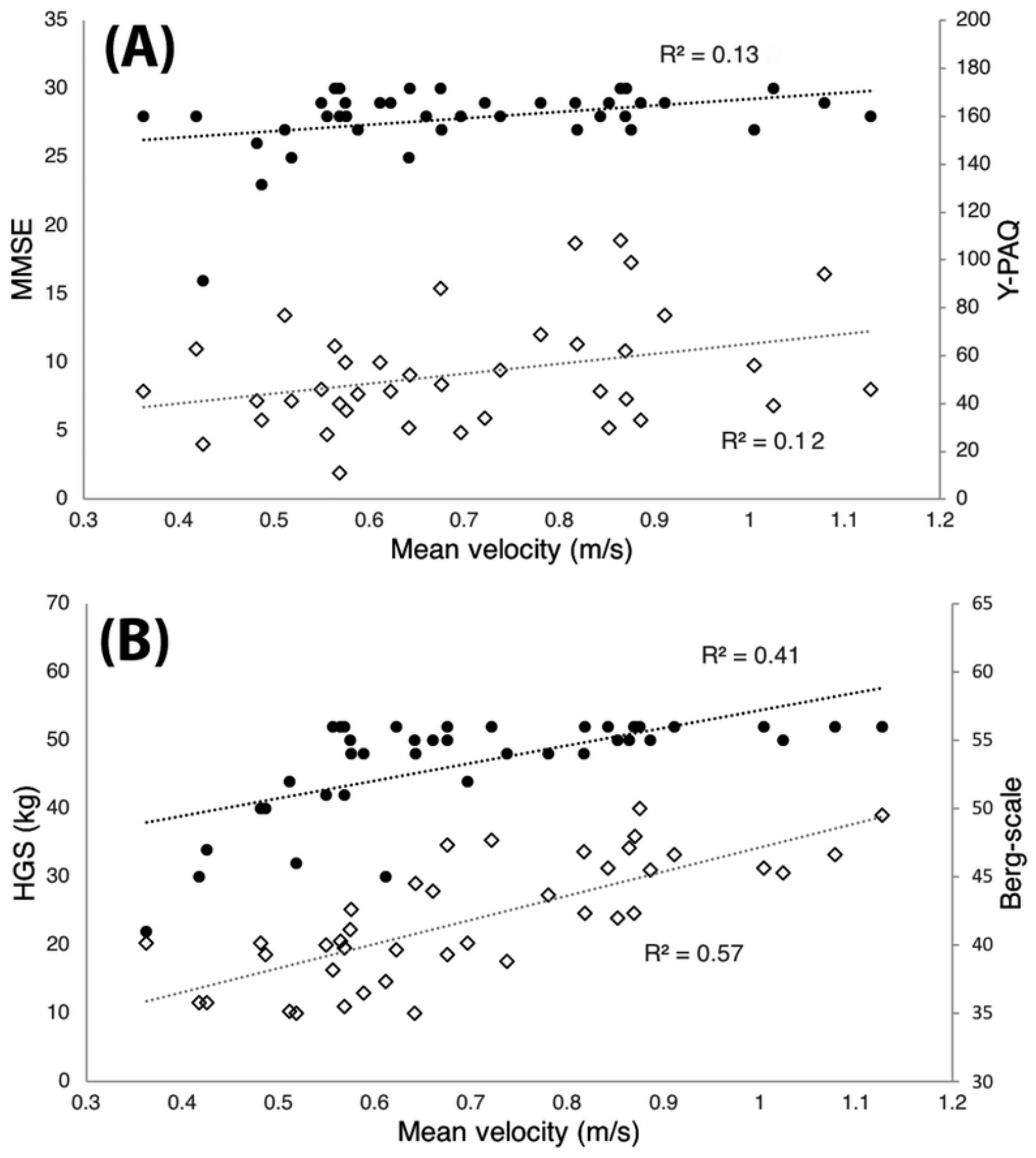
Figure 3

Figure 3

Standardized mean differences (with $90 \% \mathrm{Cl}$ ) between scores for the hand grip strength (HGS), the EQ-5D scale, the Yale Physical Activity Questionnaire (Y-PAQ), the Mini mental state examination (MMSE) and the Berg scale from the strongest (G1) and weaker (G2) participants.

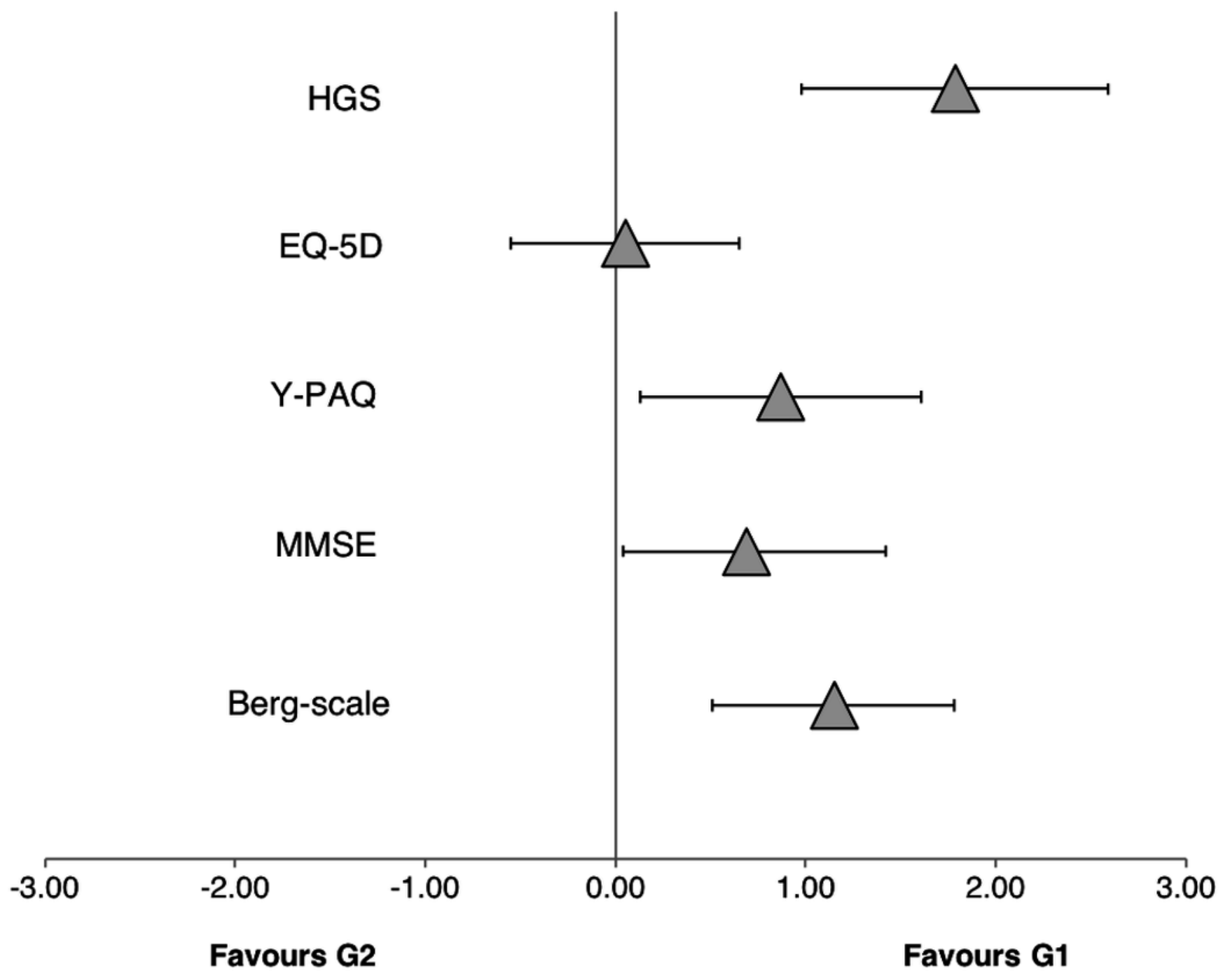

